calcium hydroxylapatite group than the collagen group $(4.0 \mathrm{ml}$ versus $6.6 \mathrm{ml}, P<0.0001)$. No serious systemic adverse events were reported in either group.

The authors conclude that calcium hydroxylapatite has efficacy and safety equivalent to bovine collagen; the main advantage of the new agent is that patients with SUI are more likely to achieve improvement with only one injection.

Original article Mayer RD et al. (2007) Multicenter prospective randomized 52-week trial of calcium hydroxylapatite versus bovine dermal collagen for treatment of stress urinary incontinence. Urology 69: 876-880

\section{Excessive multivitamin use might increase the risk of advanced and fatal prostate cancer}

Multivitamin supplements are taken by $35 \%$ of adults in the US but, alarmingly, epidemiological evidence suggests that multivitamin use might be associated with more rapid progression of prostate cancer. Other data indicate that multivitamin use may protect against initiation of the disease. In a large prospective study, Lawson et al. investigated the association between multivitamin use and the risk of localized, advanced and fatal prostate cancer in 295,344 men enrolled in the NIH-American Association of Retired Persons Diet and Health Study.

All participants were cancer free at enrollment (1995-1996), when information on use of multivitamin and individual supplements during the preceding 12 months was obtained by questionnaire. Prostate cancer was diagnosed in 10,241 participants (8,765 localized and 1,476 advanced) during 5 years follow-up. Mortality analysis (6 years of follow-up) identified 179 cases of fatal prostate cancer. After adjustment for prostate cancer risk factors, multivitamin use was not associated with the risk of localized prostate cancer. By contrast, men who reported excessive use of multivitamin supplements (more than seven times per week) had an increased risk of advanced and fatal prostate cancer compared with never users (relative risk 1.32 [95\% Cl 1.04-1.67] and 1.98 [95\% Cl 1.07-3.66], respectively); concomitant use of some individual supplements seemed to increase the risk further.

Detection bias is unlikely to have distorted the positive association found between excessive multivitamin use and advanced and fatal prostate cancer. These worrying findings require further assessment.

Original article Lawson KA et al. (2007) Multivitamin use and risk of prostate cancer in the National Institutes of HealthAARP Diet and Health study. J Natl Cancer Inst 99: 754-764

\section{No benefit of clodronate treatment in patients with locally advanced prostate cancer}

Adjuvant sodium clodronate, a first-generation bisphosphonate, showed no benefit in patients with nonmetastatic prostate cancer in a recent randomized, double-blind, placebo-controlled trial.

Mason and colleagues from the UK tested whether bisphosphonates slow the development of symptomatic bone metastases in prostate cancer patients. The study included men recently diagnosed with stage T2-T4 prostate cancer with no evidence of metastases, who had not previously undergone bisphosphonate treatment or long-term hormone therapy. A total of 508 men were randomized in a 1:1 ratio to receive $2,080 \mathrm{mg}$ of oral sodium clodronate or placebo daily for up to 5 years. Patients were evaluated at 6 weeks after randomization, then every 6 months for 2 years, and yearly thereafter. Median follow-up was 118 months.

The results showed no benefit in bonemetastasis-free survival in the clodronate group compared with the placebo group (80 events vs 68 events, hazard ratio [HR] 1.22, 95\% Cl 0.88-1.68) or overall survival (130 deaths vs 127 deaths, HR 1.02, 95\% Cl 0.80-1.30). Clodronate was generally well tolerated, although there were more adverse events reported in the clodronate group than in the placebo group $(P=0.18)$; patients receiving clodronate required dose modification more often $(P=0.002)$.

The authors conclude that clodronate should no longer be investigated in the adjuvant setting as a treatment for nonmetastatic prostate cancer, but trials of more potent bisphosphonates should be carried out, for example zoledronic acid which is being assessed in the MRC stampede trial (ISRCTN78818544).

Original article Mason MD et al. (2007) Oral sodium clodronate for nonmetastatic prostate cancer-results of a randomized double-blind placebo-controlled trial: Medical Research Council PR04 (ISRCTN61384873). J Natl Cancer Inst 99: 765-776 